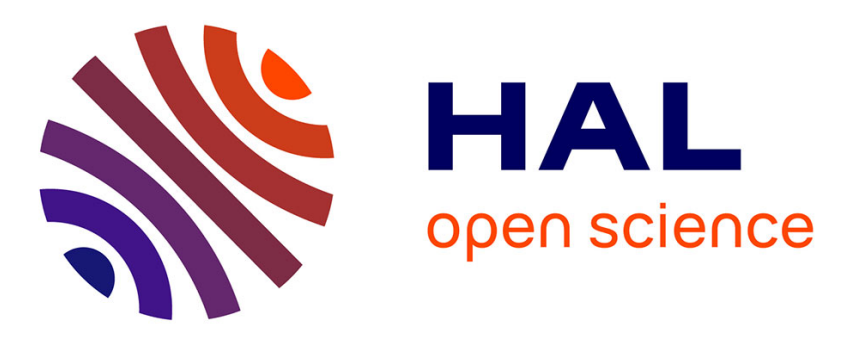

\title{
Rigidité artérielle mesurée par pOpmètre® chez les patients à risque cardiovasculaire, lien aux plaques d'athérome carotidien
}

Magid Hallab, Mathieu Collette, C. Terrier-Barbeau, Mathieu Legrand, Pierre-Henri Ducluzeau-Fieloux, Gilles Berrut, Georges Lefthériotis

\section{To cite this version:}

Magid Hallab, Mathieu Collette, C. Terrier-Barbeau, Mathieu Legrand, Pierre-Henri DucluzeauFieloux, et al.. Rigidité artérielle mesurée par pOpmètre® chez les patients à risque cardiovasculaire, lien aux plaques d'athérome carotidien. Annales de Cardiologie et d'Angéiologie, 2013, 62 (3), pp.189-192. 10.1016/j.ancard.2013.04.001 . hal-03328661

\section{HAL Id: hal-03328661 \\ https://univ-angers.hal.science/hal-03328661}

Submitted on 30 Aug 2021

HAL is a multi-disciplinary open access archive for the deposit and dissemination of scientific research documents, whether they are published or not. The documents may come from teaching and research institutions in France or abroad, or from public or private research centers.
L'archive ouverte pluridisciplinaire HAL, est destinée au dépôt et à la diffusion de documents scientifiques de niveau recherche, publiés ou non, émanant des établissements d'enseignement et de recherche français ou étrangers, des laboratoires publics ou privés. 


\title{
Rigidité artérielle mesurée par pOpmètre ${ }^{\circledR}$ chez les patients à risque cardiovasculaire, lien aux plaques d'athérome carotidien
}

\section{Regional and peripheral arterial stiffness measured by pOpmetre ${ }^{\circledR}$ in patients with Cvx risk factor, link with carotid plaques}

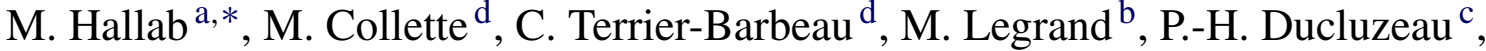 \\ G. Berrut ${ }^{\mathrm{a}}$, G. Lefthériotis ${ }^{\mathrm{b}}$ \\ a Département de médecine gériatrique, hôpital universitaire, place Alexis-Ricordeau, 44093 Nantes cedex 1, France \\ ${ }^{\mathrm{b}}$ Laboratoire d'explorations vasculaires, CHU d'Angers, 49933 Angers, France \\ ${ }^{\mathrm{c}}$ Département de diabétologie et de nutrition, hôpital universitaire d'Angers, 49000 Angers, France \\ ${ }^{\mathrm{d}}$ Laboratoire d'ingénierie et d'automatisme, université d'Angers, 49000 Angers, France
}

Reçu le 20 mars 2013 ; accepté le $1^{\text {er }}$ avril 2013

Disponible sur Internet le 19 avril 2013

\section{Résumé}

But. - La rigidité artérielle (RA) est un prédicteur indépendant de risque cardiovasculaire. Elle peut être estimée facilement par une nouvelle technique : pOpmètre ${ }^{\circledR}$ (Axelife SAS, France). Nous avons étudié le lien entre les indices pOpmètre ${ }^{\circledR}$ et (1) la présence de plaques carotidiennes, (2) la relation avec l'âge chez des patients à risque cardiovasculaire.

Méthodes. - La présence de plaques carotidiennes et l'épaisseur intima-média (EIM) ont été déterminées par écho doppler chez 77 patients à risque. La RA aortique a été évaluée par impédance-mètre (Physioflow ${ }^{\circledR}$ ESAOTE, Italie) et la VOPdo par pOpmètre ${ }^{\circledR}$, qui calcule la vitesse de l'onde de pouls (VOPdo), la différence des temps de transit (Dod), et un indice de vélocité. La pression artérielle et l'indice de pression systolique (IPS) ont été mesurés.

Résultat. - Les deux groupes (avec ou sans plaques) étaient comparables pour la pression artérielle brachiale et l'IPS (1,15 $\pm 0,04$ versus $1,12 \pm 0,03 ; \mathrm{ns})$. Le groupe avec plaques carotidiennes était plus âgé ( $59 \pm 2$ ans versus $49 \pm 2, p<0,002)$, avec EIM supérieure ( $0,69 \pm 0,02$ versus $0,63 \pm 0,01 \mathrm{~mm}, p<0,004)$. La RA par impédance $(10,4 \pm 0,7$ versus $8,2 \pm 0,5 \mathrm{~m} / \mathrm{s}, p<0,02)$ et les indices pOpmètre ${ }^{\circledR}$ (VOPdo $[14,3 \pm 1,0$ versus $10,7 \pm 0,7 \mathrm{~m} / \mathrm{s}, p<0,004]$; Dod [57,9 $\pm 5,1$ versus 73,5 $\pm 3,5 \mathrm{~ms}, p<0,01]$; indice de vélocité du pouls [IVP] [1,51 $\pm 0,3 \mathrm{versus} 1,41 \pm 0,2$, $p<0,006])$ étaient augmentés dans le groupe avec plaques. L'âge corrélait avec pression artérielle systolique (PAS) $\left(r^{2}=0,11, p=0,002\right)$, RA $\left(r^{2}=0,15, p=0,002\right)$, pOpmètre ${ }^{\circledR}$ VOPdo $\left(r^{2}=0,24, p<10^{-4}\right)$, l'EIM $\left(r^{2}=0,17, p=0,0003\right)$, pas avec l'IPS $\left(r^{2}=0,04, p=0,07\right)$.

Conclusion. - Chez des patients à risque vasculaire, la RA mesurée par pOpmètre ${ }^{\circledR}$ est associée à la présence de plaques carotidiennes. (C) 2013 Elsevier Masson SAS. Tous droits réservés.

Mots clés : Plaques carotidiennes ; Rigidité artérielle ; Facteur de risque cardiovasculaire ; Vitesse de l'onde de pouls ; Temps de transit

\section{Abstract}

Purpose. - Aortic stiffness is a functional and structural consequence of ageing and arteriosclerosis. Regional arterial stiffness can be easily evaluated using pOpmetre ${ }^{\circledR}$ (Axelife SAS, France). This new technique assesses the pulse wave transit time (TT) between the finger (TTf) and the toe (TTt). Based on height chart, regional pulse wave velocity (PWV) between the toe and the finger can be estimated (PWVtf). pOpscore ${ }^{\circledR}$ index is also calculated as the ratio between PWVtoe and PWVfinger and can be considered as a peripheral vascular stiffness index. The aim of the study was to evaluate the relationship between pOpmetre ${ }^{\circledR}$ indices and the presence of carotid plaques in a population with cardiovascular risk factors.

Methods. - In 77 consecutive patients recruited for a vascular screening for atherosclerosis (46 men aged $54 \pm 2$ years; 31 women aged $49 \pm 3$ years; ns), the difference between TTt and TTf (called Dt-f), the regional pulse wave velocity between the toe and the finger $(\mathrm{PWVtf}=$ constant $\times$ height/Dt-f $\mathrm{m} / \mathrm{s})$ and pOpscore ${ }^{\circledR}$ were measured by pOpmetre ${ }^{\circledR}$. Presence of carotid plaques was assessed using ultrasound imaging. The local aortic stiffness (AoStiff) was evaluated by the Physioflow ${ }^{\circledR}$ system.

\footnotetext{
* Auteur correspondant.

Adresse e-mail : magid.hallab@gmail.com (M. Hallab).
} 
Results. - No difference was found between patients with or without carotid plaques $(n=25$ versus 52$)$ for Ankle-Brachial Pressure Index (ABPI: $1.15 \pm 0.04$ versus $1.12 \pm 0.03$ ), nor for diastolic or systolic blood pressure $(87 \pm 3$ versus $82 \pm 2 ; 137 \pm 3$ versus $132 \pm 2 \mathrm{mmHg}$ ). The first group was older than the second ( $59 \pm 2$ versus $49 \pm 2$ years, $P<0.002)$ with a larger intimae media thickness $(0.69 \pm 0.02$ versus $0.63 \pm 0.01$ mm, $P<0.004)$, a higher AoStiff $(10.4 \pm 0.7$ versus $8.2 \pm 0.5 \mathrm{~m} / \mathrm{s}, P<0.02)$, and PWVtf $(14.3 \pm 1.0$ versus $10.7 \pm 0.7 \mathrm{~m} / \mathrm{s}, P<0.004)$ and a shorter Dt-f $(57.9 \pm 5.1$ versus $73.5 \pm 3.5 \mathrm{~ms}, P<0.01)$. PWVtf $\left(r^{2}=0.49, P<0.0001\right)$ and Dt-f $\left(r^{2}=0.54, P<0.0001\right)$ correlated with age. A significant difference in pOpscore ${ }^{\circledR}$ index was observed between both groups $(1.51 \pm 0.3$ versus $1.41 \pm 0.2, P<0.006)$.

Conclusion. - Our results show a significant arterial stiffness indices measured by pOpmetre ${ }^{\circledR}$ in patients with and without carotid plaques.

(C) 2013 Elsevier Masson SAS. All rights reserved.

Keywords: Carotid plaques; Arterial stiffness; Cardiovascular risk factor; Pulse wave velocity; Transit time

La rigidité artérielle (RA) est un témoin d'altération pariétale qui modifie les propriétés physiques de l'artère telle que la compliance artérielle. La vitesse de propagation de l'onde de pouls carotide-fémorale (VOPcf) est un bon reflet de la RA. Dans certaines populations, c'est un facteur de risque indépendant [1] qui est meilleur prédicteur d'évènements cardiovasculaires [2] que les facteurs de risque classiques (diabète, hypertension, cholestérol, tabac), voire la mortalité toute cause dans la population générale [3].

De nouveaux appareils mesurent différents indices de rigidité comme la pression centrale, l'index d'augmentation, et de nombreux autres paramètres portant sur la RA [4], certains par des fonctions mathématiques de transfert à partir de la pression artérielle étalonnée au bras. Notons que la mesure de la distance dans la VOPcf a été débattue cette dernière décennie, et un consensus a été établi pour prendre en compte $80 \%$ de la mesure directe entre le site carotidien et le site fémoral [5]. Par ailleurs, la mesure de la pression centrale, qui nécessite un étalonnage de la mesure selon la pression artérielle brachiale, ne semble pas apporter de bénéfice supplémentaire sur la mortalité par rapport à la pression brachiale.

pOpmètre ${ }^{\circledR}$ (Axelife ${ }^{\circledR}$ SAS, France) est un nouvel appareil qui mesure les temps de transit entre le doigt et l'orteil en fonction de l'onde R de l'ECG. Il a été validé par comparaison à la VOPcf [6]. Il mesure un indice (pOpscore ${ }^{\circledR}$ ) qui semble indépendant de la pression artérielle [7]. L'hypothèse clinique de cette méthode est soutenue par le fait qu'il n'existe pas de lésion d'athérosclérose au niveau des membres supérieurs (en dehors de l'insuffisance rénale dans les shunts artérioveineux), et d'un point de vue scientifique démontré, la VOP n'est pas liée à l'âge au membre supérieure alors qu'elle l'est au niveau de l'aorte et des membres inférieurs [8].

Le but de ce travail est d'évaluer l'apport des indices pOpmètre ${ }^{\circledR}$ chez des patients à risque en présence ou non de plaques carotidiennes, et les liens de ces indices de rigidité avec l'âge.

\section{Méthodes}

Cette étude descriptive et prospective a été réalisée dans le service d'exploration vasculaire, au centre hospitalouniversitaire d'Angers.

Tous les patients adressés en consultation étaient informés de l'intérêt de ce travail, et ont tous donné leur consentement. Tous les paramètres nécessaires à cette étude ont été notifiés en une seule visite médicale, nous n'avons exclu aucun patient, quelle que soit la thérapeutique en cours.

\subsection{Déterminations}

Pour chaque patient inclus, le poids, la taille, et la pression artérielle mesurée après dix minutes de repos en décubitus dorsal avec un appareil automatique type Dynamap ${ }^{\circledR}$ ont été recensés.

\subsection{Mesure de la rigidité artérielle pOpmètre ${ }^{\circledR}$}

En position allongée et après dix minutes de repos, les capteurs ont été positionnés et une mesure sur 20 secondes a été effectuée. Trois indices pOpmètre ${ }^{\circledR}$ ont été calculés :

- la différence (Dod) entre le temps de transit à l'orteil (TTo) et le temps de transit au doigt (TTd);

- basé sur une charte en fonction de la taille, nous avons calculé la VOP $=$ taille $\times \mathrm{k} / \mathrm{Dod}, \mathrm{m} / \mathrm{s}$. L'utilisation d'un coefficient à la taille a été motivé par le fait que les sigmoïdes aortiques sont associées à un repère anatomique stable qu'est l'os hyoïde, et que la taille de l'arc aortiques est associée à la taille des patients [9];

- le pOpscore ${ }^{\circledR}$ étant le rapport entre la VOP au membre inférieur divisé par la VOP au membre supérieur (= VOPo/VOPd sans unité). Voir synoptique Fig. 1.

En comparaison, nous avons utilisé différentes méthodes reconnues de dépistage de l'athérosclérose, et de l'artériosclérose :

- le repérage des plaques carotidiennes et la mesure de l'épaisseur intima-média (EIM) qui ont été effectués par un opérateur entraîné à l'aide d'un appareil d'échographie usuel. Ce qui a permis de réaliser deux groupes : avec et sans plaques d'athérome ;

- mesure de la rigidité aortique : la rigidité aortique locale (AoStiff) a été mesurée par un système d'impédance [10] validé (Eaorta ${ }^{\circledR}$, Manatec Biomédical, France);

- indice de pression systolique (IPS) : 1'IPS est mesuré comme un marqueur connu de risque cardiovasculaire. Il a été mesuré avec un sphygmomanomètre au bras et à la cheville, et le premier bruit de Korotkof a été retenu par l'opérateur au moyen d'ultrasons Doppler. 


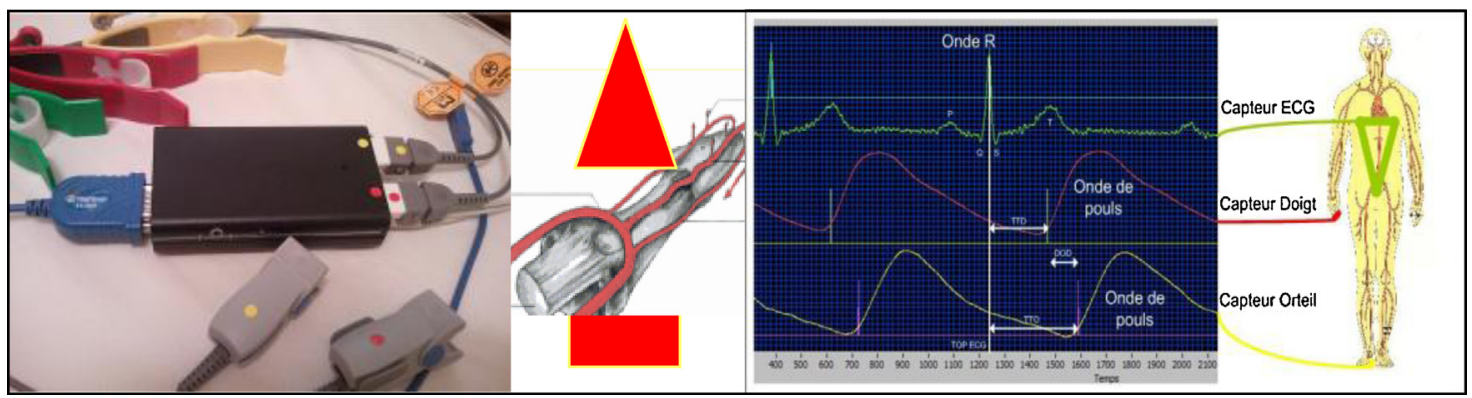

Fig. 1. Synoptique de fonctionnement de pOpmètre ${ }^{\circledR}$ : à partir de l'onde R de l'ECG, l'appareil mesure la différence de temps au pied de l'onde de l'orteil ou du doigt. Il calcule la VOP en fonction de la taille du patient divisée par la différence des temps de transit.

\subsection{Méthodes statistiques}

La comparaison de moyennes en analyse de variance et le calcul du coefficient de corrélation ont été effectués. Une analyse multivariée pas à pas a été effectuée.

\section{Résultats}

Soixante-dix-sept patients consécutifs présentant des facteurs de risque cardiovasculaire ont ainsi été recrutés. Quarantecinq hommes et 32 femmes âgés de $54 \pm 2$ versus $50 \pm 2$ ans, (moyenne \pm SEM, ns) avec un indice de masse corporelle élevé $\left(29,7 \pm 1,4\right.$ versus $\left.29,2 \pm 0,9 \mathrm{~kg} / \mathrm{m}^{2}, \mathrm{~ns}\right)$ ont été évalués. Vingt-trois d'entre eux présentaient une hypercholestérolémie, 22 étaient des fumeurs, 26 hypertendus, neuf avaient un diabète de type 2 , et 16 présentaient un pseudoxanthome élastique.

Les patients présentant des plaques carotidiennes $(n=25)$ étaient plus âgés ( $58 \pm 2$ versus $49 \pm 2$ ans ; $p<0,002)$ avec une plus grande EIM $(0,69 \pm 0,02$ versus $0,63 \pm 0,01 ; p<0,004)$ et une rigidité aortique AoStiff plus importante (10,4 $\pm 0,7$ versus $8,2 \pm 0,5 p<0,02)$. Ceux avec plaques présentaient une Dod plus courte $(57,9 \pm 5,1$ versus $73,5 \pm 3,5 \mathrm{~ms} ; p<0,01)$, avec un plus grand pOpscore ${ }^{\circledR}(1,51 \pm 0,03$ versus $1,41 \pm 0,02$ sans unités; $p<0,006)$ et une plus grande VOP pOpmètre $(14,3 \pm 1,0$ versus $10,7 \pm 0,7 \mathrm{~m} / \mathrm{s} ; p<0,004)$; aucune différence entre les deux groupe n'a été observée pour l'IPS $\left(r^{2}=0,04, p=0,07\right)$, ou pour la pression artérielle systolique (PAS) ou diastolique (Tableau 1).

L'âge était corrélé avec la RA mesurée par pOpmètre ${ }^{\circledR}$ : VOP $\left(r^{2}=0,24, p<10^{-4}\right)$, Dod $\left(r^{2}=0,28, p<10^{-4}\right)$, et pOpscore ${ }^{\circledR}$ $\left(r^{2}=0,19, p<10^{-4}\right)$, de même qu'avec l'EIM $\left(r^{2}=0,17\right.$, $p=0,0003)$, et la RA mesurée par impédance AoStiff $\left(r^{2}=0,15\right.$, $p=0,002)$. Aucune corrélation n'a été notée dans cette population entre l'âge et l'IPS $\left(r^{2}=0,04, p=0,07\right)$, mais une plus faible corrélation a été observée avec la pression artérielle systolique $\left(r^{2}=0,11 ; p<0,01\right)$ et diastolique $\left(r^{2}=0,09 ; p<0,01\right)$ (Tableau 1).

Notons enfin que la VOP mesurée par pOpmètre ${ }^{\circledR}$ était supérieure chez les hommes $(12,08 \pm 0,77$ versus $10,45 \pm 0,9 \mathrm{~m} / \mathrm{s}$; $p=0,05$ ).

Une analyse multivariée pas à pas, après ajustement par l'âge, le sexe, la PAS, pression artérielle diastolique (PAD), versus, EIM, VOPdo, montre les probabilités de signification suivantes : pour $\mathrm{EIM}=0,005$, VOPdo $=0,012$, et la pression pulsée à 0,09 .
Tableau 1

Paramètres mesurés de la population.

\begin{tabular}{llll}
\hline & \multicolumn{2}{l}{ Plaques carotidiennes } & $p$ \\
\cline { 2 - 3 } & Présence & Absence & \\
\cline { 2 - 3 } & $n=25$ & $n=52$ & \\
\hline Indices pOpmètre $^{\circledR}$ & & & \\
$\quad$ Dod (ms) & $57,9 \pm 5,1$ & $1,41 \pm 0,02$ & $<0,006$ \\
pOpscore & & $1,41(0,02)$ & $<0,006$ \\
VOP $(\mathrm{m} / \mathrm{s})$ & $14,3(1,0)$ & $10,7 \pm 0,7$ & $<0,004$ \\
AoStiff & $10,4(0,7)$ & $8,2(0,5)$ & $<0,02$ \\
EIM $(\mathrm{mm})$ & $0,69(0,02)$ & $0,63(0,01)$ & $<0,004$ \\
PAS $(\mathrm{mmHg})$ & $137,0(3,4)$ & $132,0(2,4)$ & $\mathrm{ns}$ \\
PAD $(\mathrm{mmHg})$ & $87,0(2,7)$ & $82,0(1,9)$ & $\mathrm{ns}$ \\
IPS & $1,15(0,04)$ & $1,12(0,03)$ & $\mathrm{ns}$ \\
\hline
\end{tabular}

Dod : différence des temps de transit ; AoStiff : rigidité aortique locale; EIM : épaisseur intima-média; IPS : indice de pression systolique; PAS : pression artérielle systolique; PAD : pression artérielle diastolique.

\section{Discussion}

L'IPS bas est associé à une augmentation du risque d'AVC et de maladie coronaires [11]. Des cohortes de taille moyenne (Cardiovascular Health Study, Strong Heart Study, Cohorte ARIC, etc.) ont montré sa valeur pronostique pour la mortalité cardiovasculaire [12].

Dans cette étude, les patients présentant un facteur de risque cardiovasculaire n'avaient pas de différence pour l'IPS en présence ou en l'absence de plaques carotidiennes $(p=0,07)$ le nombre de patient (77), et la puissance du test n'ont pu mettre en évidence une différence vraisemblable. Rappelons que ces plaques carotidiennes ont été confirmées par échographie et une mesure de l'EIM était supérieure dans le cas de présence de plaques. Les deux groupes étaient différents pour les paramètres de la rigidité aortique Eaorta ${ }^{\circledR}$ et la RA mesurée par pOpmètre ${ }^{\circledR}$. Seul L'EIM et la VOPdo restent probables après ajustement.

Plusieurs études démontrent que la maladie artérielle infraclinique prédit le risque d'évènements cardiovasculaires. Par exemple, une paroi carotidienne épaissie à l'échographie multiplie par deux le risque d'événement coronarien dans les dix ans qui viennent, alors que la présence de plaque carotidienne le multiplie par quatre, et la présence de sténose par huit [13].

La Fig. 2 représente les résultats de l'EIM et de la VOP mesurée par pOpmètre ${ }^{\circledR}$ pour les deux groupes. La dispersion est pratiquement identique, et le chevauchement des valeurs 

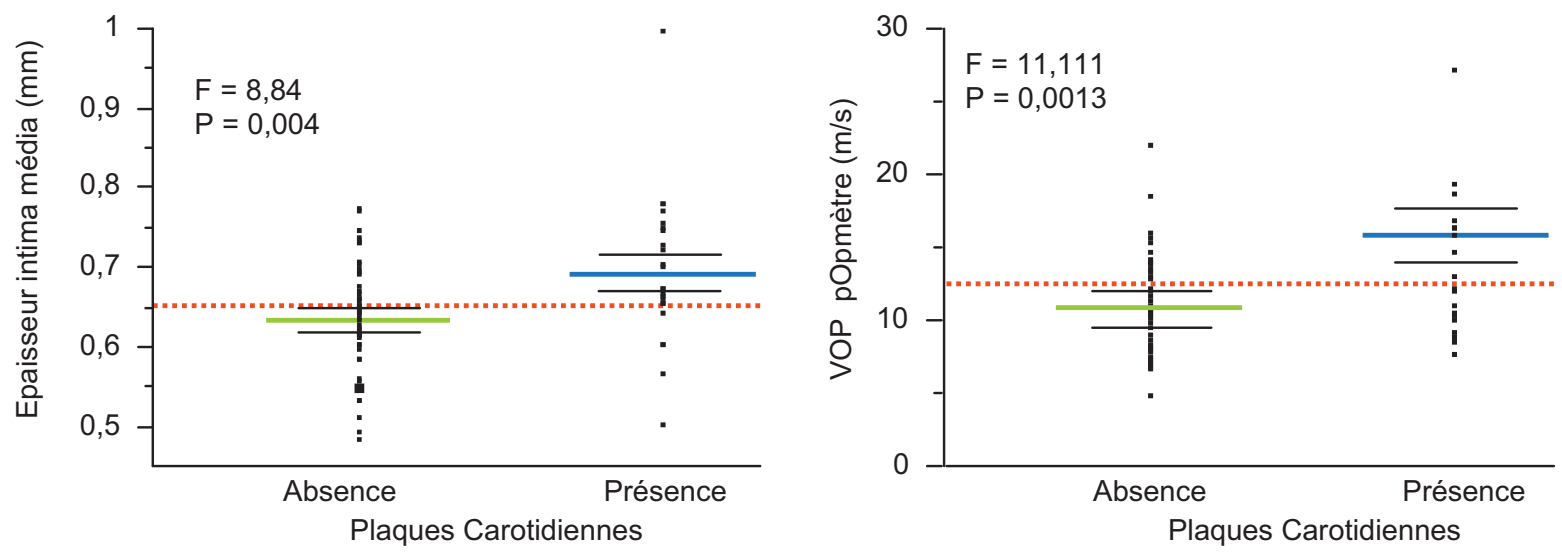

Fig. 2. Moyenne des mesures de l'épaisseur intima-média à gauche et celles de la VOP pOpmètre ${ }^{\circledR}$ en fonction de la présence ou non de plaques carotidiennes, avec les moyennes et \pm déviation standard. Les traits horizontaux en pointillé représentent la moyenne générale.

pathologiques pour les deux groupes de patients avec ou sans plaques semble lui aussi peu différent entre ces deux techniques de mesure de la RA.

Aussi, nous pouvons noter que les indices pOpmètre ${ }^{\circledR}$ sont discriminant et d'un intérêt pertinent dans l'évaluation du risque vasculaire.

pOpmètre ${ }^{\circledR}$ a été plus pertinent que l'IPS, aussi pertinent que la mesure de l'EIM pour distinguer les lésions infracliniques de l'athérosclérose. Ses indices de RA dans le système vasculaire périphérique sont liés à l'athérosclérose infraclinique, et sont en lien avec l'âge.

\section{Déclaration d'intérêts}

Le Dr Hallab déclare détenir un brevet exploité par la société Axelife SAS.

\section{Références}

[1] Laurent S, Boutouyrie P, Asmar R, et al. Aortic stiffness is an independent predictor of all-cause and cardiovascular mortality in hypertensive patients. Hypertension 2001;37:1236-41.

[2] Van Bortel LM, Laurent S, et al. Expert consensus document on the measurement of aortic stiffness in daily practice using carotid-femoral pulse wave velocity. J Hypertens 2012;3:445-8.

[3] Vlachopoulos C, Aznaouridis K, Stefanadis C. Prediction of cardiovascular events and all-cause mortality with arterial stiffness: a systematic review and meta-analysis. J Am Coll Cardiol 2010;55:1318-27.
[4] Boutouyrie P, Briet M, Collin C, Vermeersch S, Pannier B. Assessment of pulse wave velocity. Artery Res 2009;3:3-8.

[5] Huybrechts SAM, Devos DG, Vermeersch SJ, et al. Carotid to femoral pulse wave velocity: a comparison of real travelled aortic path lengths determined by MRI and superficial measurements. J Hypertens 2011;29: $1577-82$.

[6] Zhang J, Bozec E, Hallab M, Boutouyrie P, Laurent S. Étude de validation d'un nouveau dispositif de mesure de la rigidité artérielle : le pOpmètre ${ }^{\circledR}$. AMC Pratique 2011;S3:93.

[7] Hallab M, Pichierri S, Boin JM, Tramblay M, Chevalet P, Berrut G. A new index to evaluate arterial ageing independently of arterial blood pressure. Ann Cardiol Angeol 2012;61:184-7.

[8] van der Heijden-Spek JJ, Staessen JA, Fagard RH, Hoeks AP, Struijker Boudier HA, Van Bortel LM. Effect of age on brachial artery wall properties differs from the aorta and is gender dependent. Hypertension 2000;35:637-42.

[9] Hallab M, Dahou A, Chevalet P. Does the aortic valve correspond to a stable anatomic landmark? Artery Res 2011;5(4):189-90.

[10] Collette M, Humeau A, Chevalier C, Hamel JF, Leftheriotis G. Assessement of aortic stiffness by local and regional methods. Hypertens Res 2011;34:578-83.

[11] Ankle Brachial Index CollaborationFowkes FG, Murray GD, Butcher I, et al. Ankle brachial index combined with Framingham Risk Score to predict cardiovascular events and mortality: a meta-analysis. JAMA 2008;300:197-208.

[12] Criqui MH, Ninomiya JK, Wingard DL, et al. Progression of peripheral arterial disease predicts cardiovascular disease morbidity and mortality. J Am Coll Cardiol 2008;52:1736-42.

[13] Chironi G, Simon A. Facteurs de risque cardiovasculaire et prévention. Rev Prat 2010;60:1303-9. 